\title{
Oculodentodigital dysplasia and type III syndactyly: separate genetic entities or disease spectrum?
}

\author{
L A Brueton, S M Huson, B Farren, R M Winter
}

\begin{abstract}
A family is described with type III syndactyly and facies resembling the oculodentodigital dysplasia facial phenotype in the absence of any of the other characteristic findings of the latter condition. The relationship between type III syndactyly and oculodentodigital dysplasia is discussed.
\end{abstract}

Bilateral syndactyly of the fourth and fifth fingers (type III syndactyly) has been reported both as an isolated malformation and as the characteristic digital anomaly found in oculodentodigital dysplasia. Isolated type III syndactyly and oculodentodigital dysplasia have been considered distinct genetic disorders. We report a family with syndactyly of fingers 4 and 5 and facial features similar to those described in oculodentodigital dysplasia but with none of the other manifestations characteristic of the latter condition. The pedigree is shown in fig 1 .

\section{Case reports}

The proband, IV.1, was born at term after an

The Kennedy Galton Centre, Clinical Research Centre, Northwick Park Hospital, Watford Road, Harrow, Middlesex HAI 3UJ.

L A Brueton, S M Huson, B Farren, $\mathbf{R} \mathbf{M}$ Winter Correspondence to Dr Brueton.

Received for publication 17 May 1989.

Revised version accepted for publication 5 September 1989.

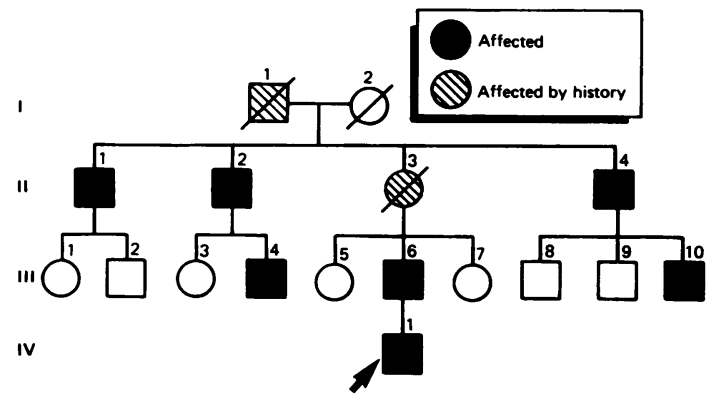

Figure 1 Pedigree. uneventful pregnancy. At birth he was noted to have bilateral syndactyly of the fourth and fifth fingers (fig 2). Physical examination was otherwise normal. In the

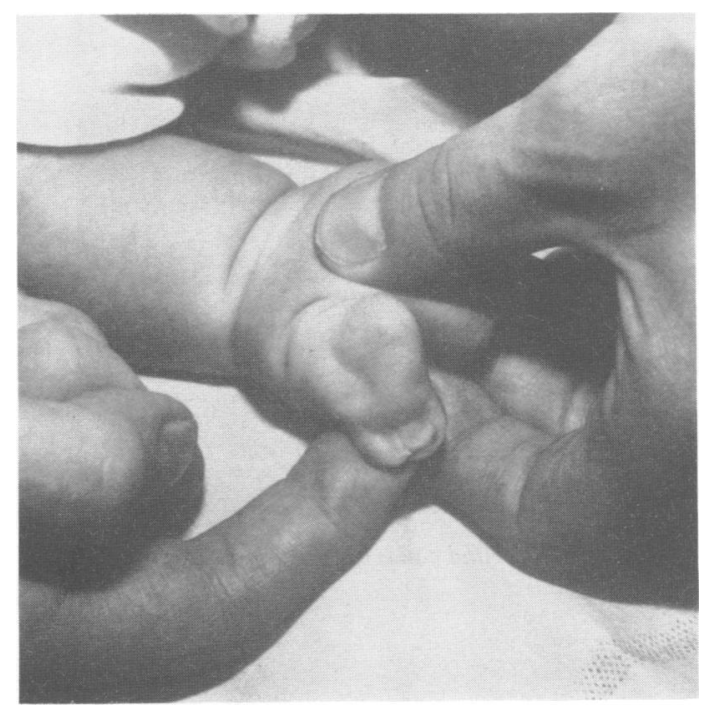

Figure 2 The right hand of IV.1 showing syndactyly of the fourth and fifth fingers.

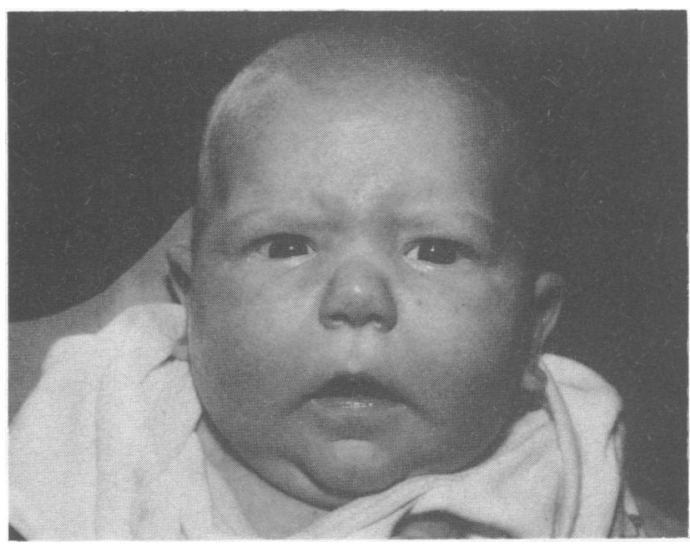

Figure 3 Facial appearance of the proband, IV.1, aged 2 months. 
family history eight other members were known to have had similar abnormalities (fig 1).

On examination at 10 months of age, there was bilateral complete syndactyly of fingers 4 and 5 with

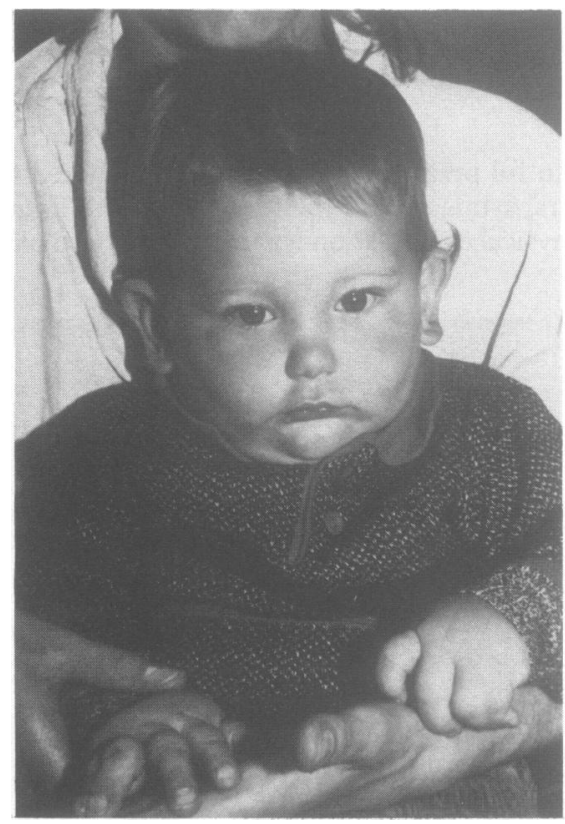

Figure 4 The face and hands of IV.I aged 13 months. fourth finger camptodactyly. The feet were normal. The head shape was brachycephalic (cephalic index $81.4)$ with a normal head circumference $(48.3 \mathrm{~cm}$, 90th centile). Although he appeared to have a broad nasal bridge, the inner canthal, interpupillary, and outer canthal distances were all less than the 3rd centile. There were no auditory, ocular, or developmental problems and no abnormality of hair growth. $\mathrm{He}$ had eight teeth all of which were normal. Fig 3 shows his facial appearance at 2 months and fig 4 his face and hands at 13 months of age. He has since had surgical correction of the syndactyly. Preoperative hand radiographs taken aged 18 months show bilateral $4 / 5$ syndactyly with bony fusion of the terminal phalanges of the fourth and fifth fingers, associated camptodactyly, and absence of the middle phalanx of the fifth finger bilaterally (fig 5).

The clinical details of IV.1 are summarised in the table together with the findings in the other affected family members. Nobody examined had enamel hypoplasia of the teeth, significant dental caries, or any abnormality of hair growth. All of the affected family members had normal palates and normal feet. Fig 6 shows the facies and hands of the other living affected family members.

III.2, III.7, III.8, and III.9 were examined and found to be normal. None had syndactyly and their facial features (fig 7) differed from those of affected family members. Subject III.9 had a long nose with a narrow bridge but the other features characteristic of the ODD facial phenotype were absent and his hands and feet were completely normal.

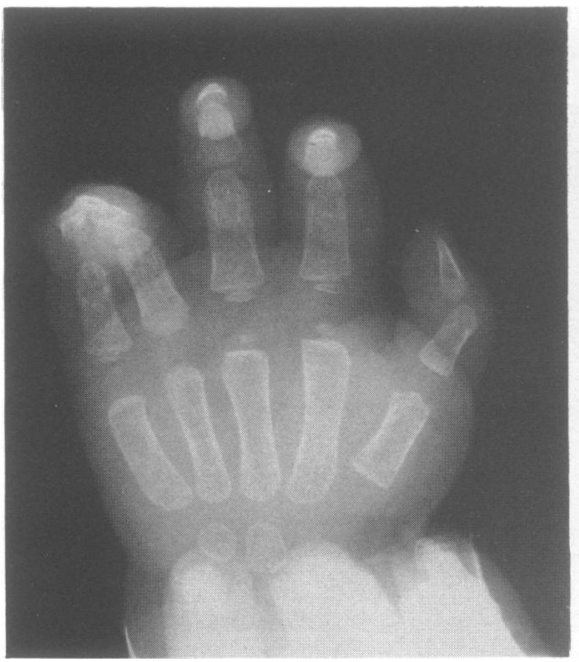

(a)

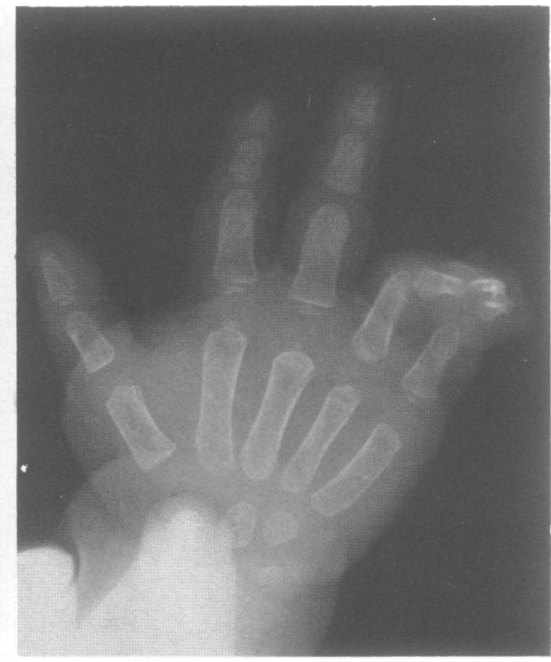

(b)
Figure 5 Radiographs of (a) left and (b) right hands of $I V .1$ aged 18 months. 


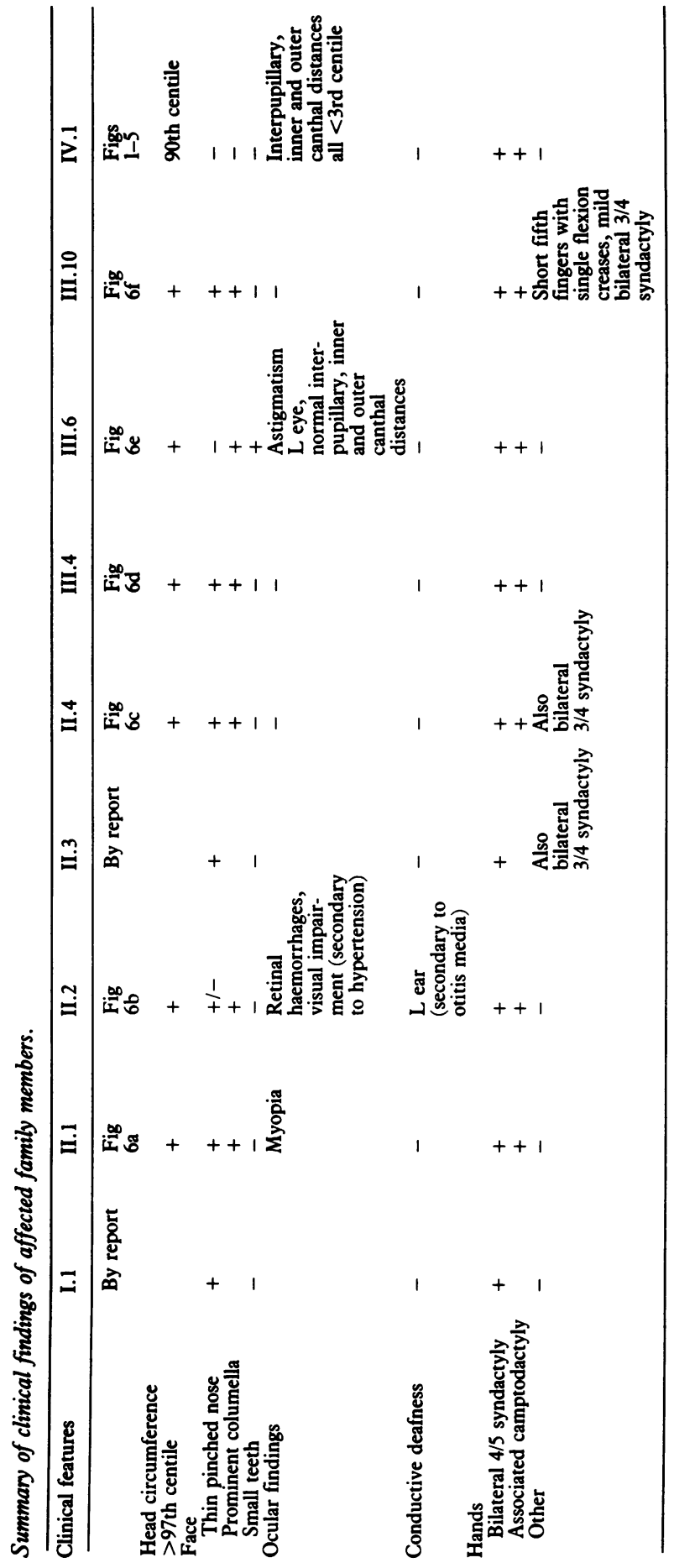



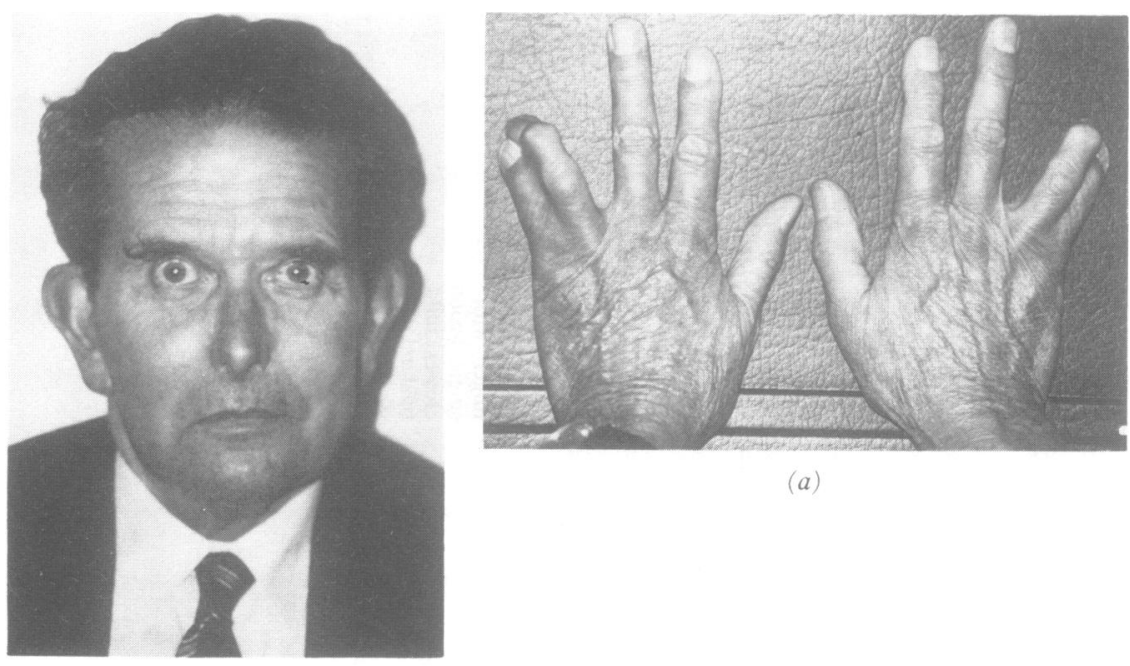

Figure 6 The facies and hands of the other living affected family members:

(a) $I I .1,(b) I I .2$, (c) II.4, (d) III.4, (e) III.6, (f) III. 10 .
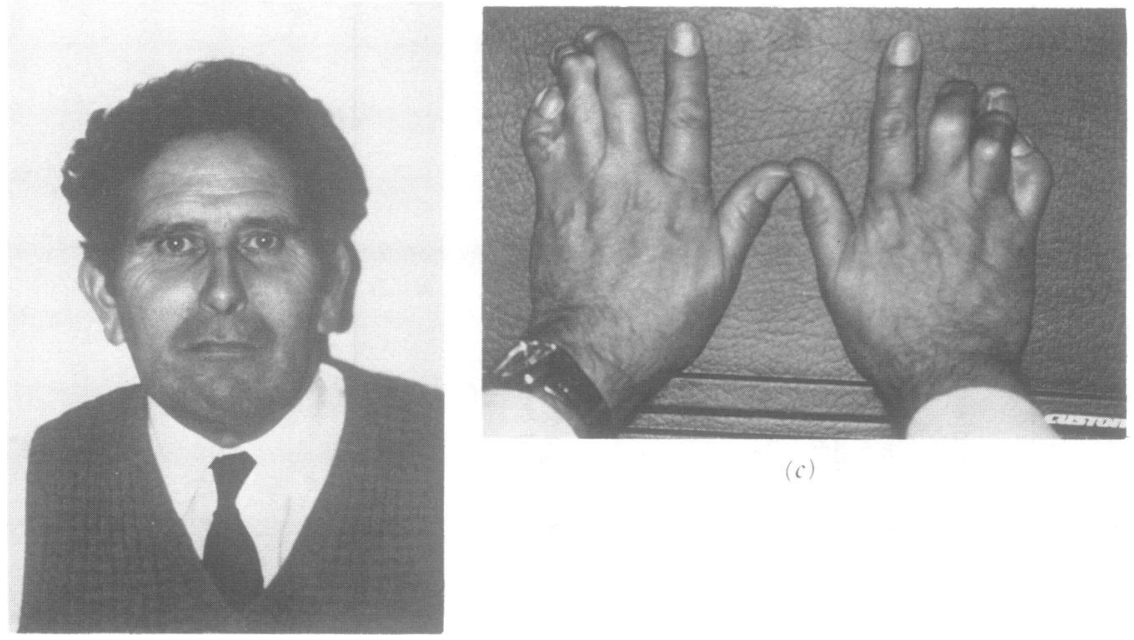

(c) 

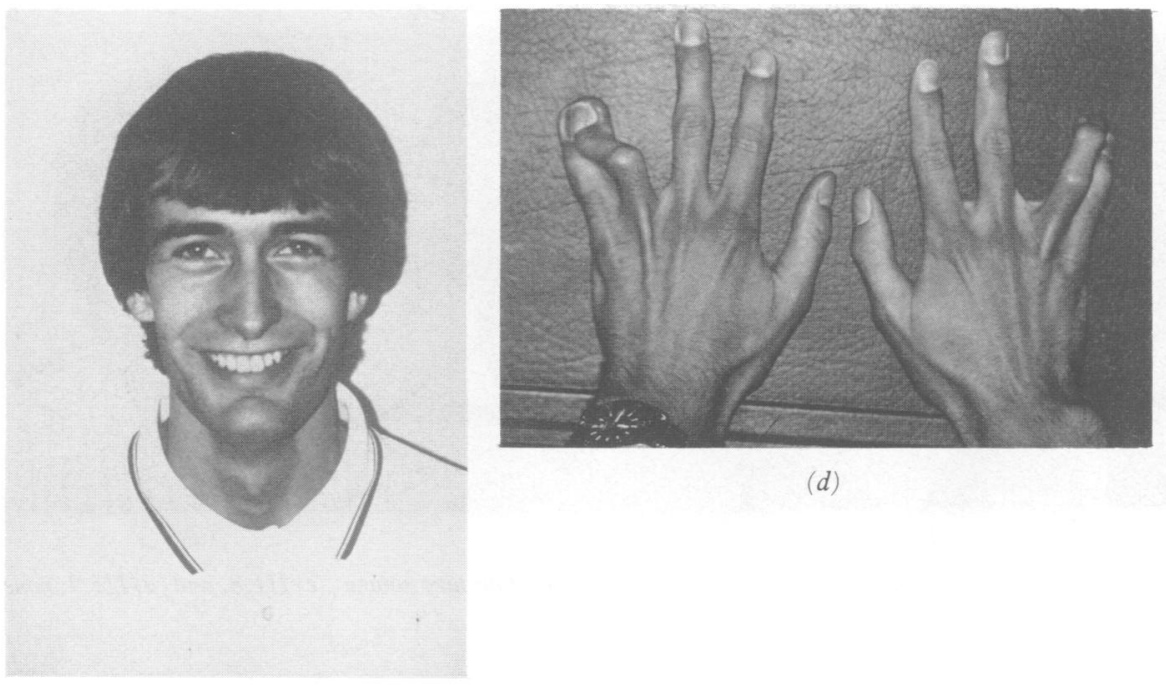

(d)
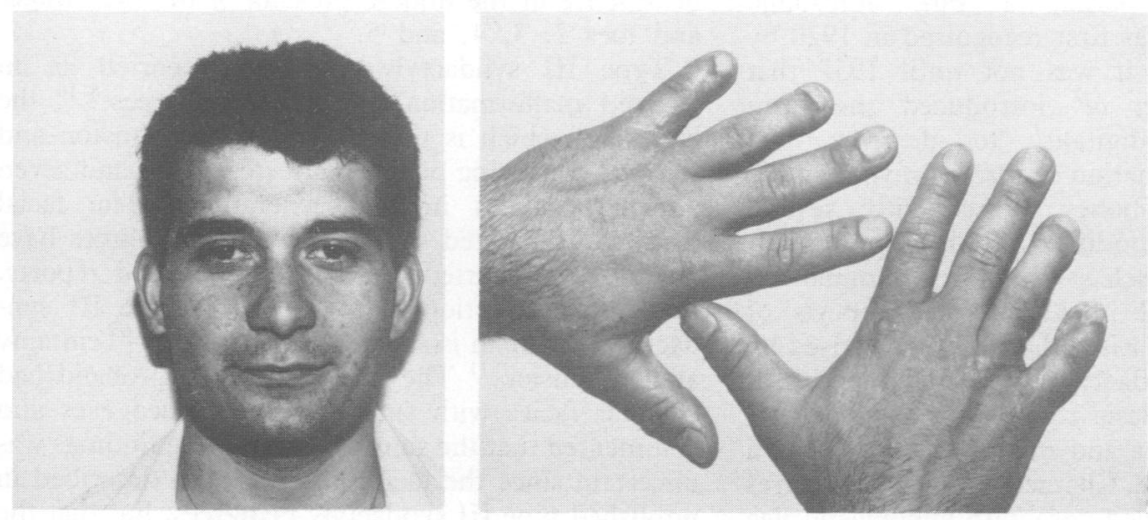

(e)
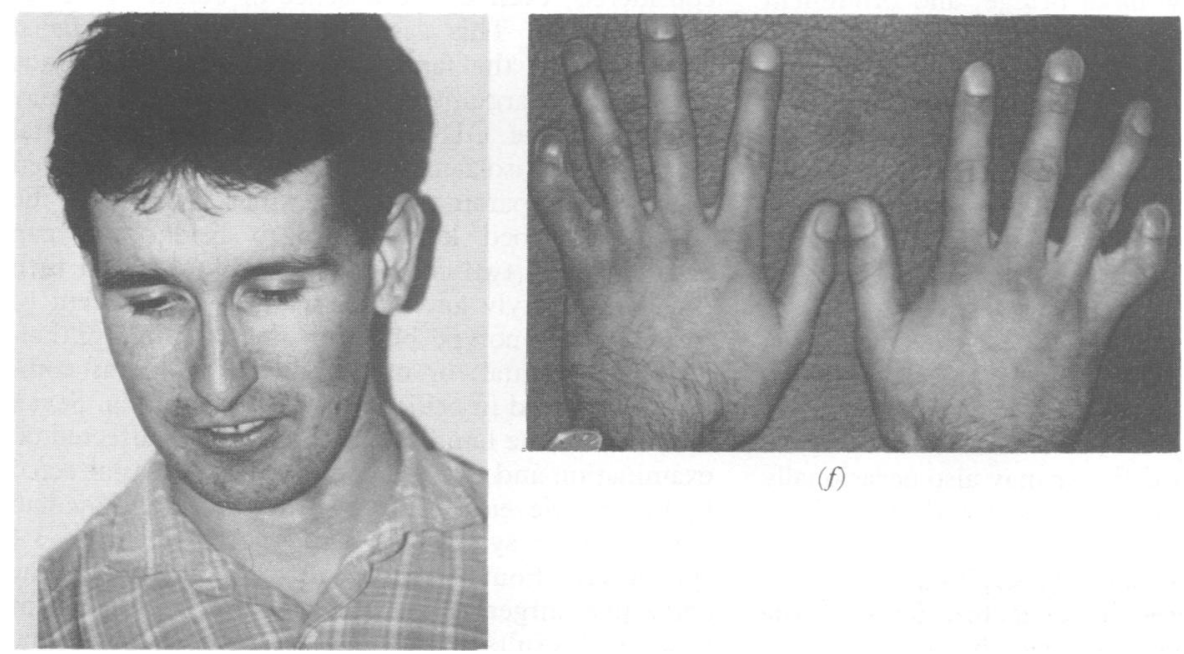

$(f)$ 


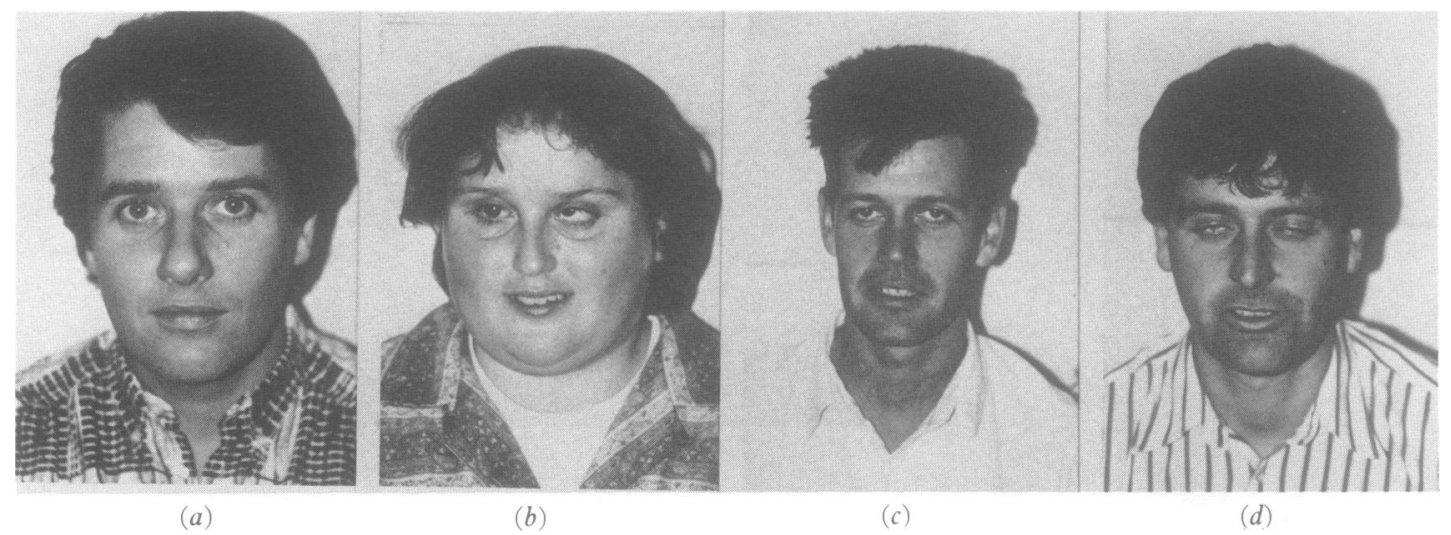

Figure 7 The facies of $(a) I I I .2,(b) I I I .7$, who has had previous neurosurgery for a pituitary tumour, (c) III.8, and (d) III.9, none of whom is affected.

\section{Discussion}

Oculodentodigital dysplasia, a rare autosomal dominant condition, was first recognised in 1920 by Lohmann. ${ }^{1}$ However, it was not until 1957 that Meyer-Schwickerath et $a l^{2}$ introduced the term 'dysplasia oculodentodigitalis' to describe two patients with a malformation syndrome involving the eyes, nose, teeth, and bones. Subsequently, several articles reviewing this condition have been published, including that by Judisch et $a l^{3}$ which summarised the findings described in 41 accepted reports of oculodentodigital dysplasia. ODD is characterised by syndactyly and camptodactyly of the fourth and fifth fingers, enamel hypoplasia resembling amelogenesis imperfecta, microcornea and microphthalmos, and a typical facial appearance. Characteristic facial features include a narrow, pinched nose with hypoplastic alae nasi, prominent columella and thin anteverted nares together with a narrow nasal bridge, and prominent epicanthic folds giving the impression of hypertelorism (although often not confirmed by measurement $^{4}$ ). The hair may be thin and sparse, the teeth small and carious, and the head circumference somewhat reduced. Eye defects consist of microcornea, iris anomalies, and in some cases secondary glaucoma, small palpebral fissures, persistent pupillary membrane, and microphthalmos. The pupil may be eccentric. Both conductive deafness secondary to secretory otitis media and cleft palate have been reported in several persons with ODD. Bilateral complete syndactyly of the fourth and fifth fingers (type III syndactyly) is the characteristic digital malformation. The third finger may also occasionally be involved and associated camptodactyly is a common finding.

Radiographs typically show hyperplasia of the body of the mandible and alveolar ridges, broadening of the tubular bones, bilateral syndactyly and campto- dactyly of the fourth and fifth fingers, and hypoplasia or absence of the middle phalanx of the fifth finger and toes $2,3,4$, and $5 . .^{-7}$

Type III syndactyly has been reported as an isolated malformation in several pedigrees, ${ }^{8-10}$ the largest of which is that described by Johnston and Kirby ${ }^{10}$ consisting of seven affected males and seven affected females in five generations. The facial features of affected subjects in these pedigrees have never been reported. Reviewing published reports, the only description of the facies in type III syndactyly was in an isolated case reported by Temtamy and McKusick. ${ }^{11}$ They noted that the proband had peculiar facies with small, widely spaced eyes and commented that the significance of these findings was uncertain since the facies had not been described in published type III syndactyly pedigrees, but that the diagnosis of oculodentodigital dysplasia had to be considered, even in the absence of ocular or dental abnormalities. This case shows considerable facial similarities to the family reported here, and lends weight to the argument that type III syndactyly may be part of the ODD spectrum. Oculodentodigital dysplasia and isolated type III syndactyly have been considered separate genetic entities. However, the family described here seems to bridge the gap between these two conditions, with fourth and fifth finger syndactyly and facial features reminiscent of the ODD phenotype but with none of the ophthalmological, dental, or other skeletal problems commonly reported in oculodentodigital dysplasia. Seven members of the family were confirmed as affected on examination and two others were reported as affected by history. Seven had typical $4 / 5$ syndactyly, one had marked $3 / 4 / 5$ syndactyly, and one, by history, $3 / 4$ syndactyly. Four of those affected had undergone corrective surgery for the syndactyly with excellent functional results in each case. The consistency of the 
facial appearance in all the affected adults of the family described is striking, although the features typical of ODD are not as yet clearly obvious in the 10 month old proband. This is in keeping with the pattern of development of the ODD facial phenotype discussed in the paper by Patton and Laurence, ${ }^{12}$ which illustrated obvious characteristic features by the fourth year of life in subjects with ODD, their presence in infancy only being identified in retrospect.

Small head circumference has been reported as an occasional finding in ODD..$^{513}$ However, all the affected family members examined in this study had head circumferences $>90$ th centile. All of the affected subjects seen had normal hair and teeth. None had any of the ocular problems known to be associated with ODD or any other obvious ophthalmological abnormality on routine examination. One member had unilateral hearing loss secondary to otitis media.

In conclusion, we describe a kindred with type III syndactyly segregating in an autosomal dominant fashion over four generations. The facial features of affected persons clearly resemble those described in the oculodentodigital syndrome; however, none had any of the ocular or dental anomalies commonly associated with this disorder. The digital anomalies in type III syndactyly are identical and although the facial phenotype in ODD is considered pathognomonic, the facial features have not been described in the published pedigrees with type III syndactyly and may have been overlooked. It seems possible that these two conditions either form part of a contiguous gene syndrome, the phenotypic variation being explained by the loss of function of varying amounts of genetic material, or alternatively they may be allelic disorders. The consistency of clinical findings within our family makes variable expression of a single gene unlikely.

We are grateful to Mrs S Kingsley for typing the manuscript. This work was supported by the Medical Research Council.

1 Lohmann W. Beitrag zur Kenntnis des reinen Mikrophthalmus. Arch Augenheilkd 1920;86:136-41.

2 Meyer-Schwickerath G, Gruterich E, Weyers H. Mikrophthalmus syndrome. Klin Monatsbl Augenheilkd 1957;131:18-30.

3 Judisch GF, Martin-Casals A, Hanson JW, Olin WH. Oculodentodigital dysplasia: four new reports and a literature review. Arch Ophthalmol 1979;97:878-84.

4 Fara M, Gorlin RJ. The question of hypertelorism in oculodentoosseous dysplasia. Am $\mathcal{F}$ Med Genet 1981;10:101-2.

5 Reisner SH, Kott E, Bornstein B, Salinger H, Kaplin J, Gorlin RJ. Oculodentodigital dysplasia. Am $\mathcal{F}$ Dis Child 1969;118: 600-7.

6 Kurlander GJ, Lowy NW, Campbell JA. Roentgen differentiation of oculodentodigital syndrome and the Hallermann-Streiff syndrome in infancy. Radiology 1966;86:77-85.

7 Rajic DS, De Veber Li. Hereditary oculodentoosseous dysplasia. Ann Radiol 1966;9:224-31.

8 Bell $\mathrm{J}$. Three further cases of hereditary digital anomaly seen in the out-patient department of Great Ormond Street Hospital for Sick Children. Ann Eugen 1931;4:233-7.

9 Collette AT. A case of syndactylism of the ring and little fingers. Am f Hum Genet 1954;6:241-3.

10 Johnston $\mathrm{O}$, Kirby VV. Syndactyly of the ring and little finger. Am f Hum Genet 1955; 7:80-2.

11 Temtamy SA, McKusick VA. The genetics of hand malformations. Birth Defects 1978;14(3):309-14.

12 Patton MA, Laurence KM. Three new cases of oculodentodigital (ODD) syndrome: development of the facial phenotype. $\mathcal{f}$ Med Genet 1985;22:386-9.

13 Sugar HS, Thompson JP, Davis JD. The oculodento-digital dysplasia syndrome. Am $\mathcal{f}$ Ophthalmol 1966;61:1448-51. 